\title{
Sentinel lymph node in vulvar cancer
}

\author{
Agnieszka Rychlik, Mariusz Bidzinski, Jakub Rzepka, Szymon Piatek^^
}

Department of Gynecologic Oncology, Maria Skłodowska-Curie National Research Institute of Oncology, Warsaw, Poland

Contributions: (I) Conception and design: M Bidzinski; (II) Administrative support: S Piatek; (III) Provision of study materials or patients: A Rychlik; (IV) Collection and assembly of data: A Rychlik, J Rzepka, S Piatek; (V) Data analysis and interpretation: All authors; (VI) Manuscript writing: All authors; (VII) Final approval of manuscript: All authors.

Correspondence to: Szymon Piatek, MD, PhD. Department of Gynecologic Oncology, Maria Skłodowska-Curie National Research Institute of Oncology, 5 Roentgena Street, 02-781 Warsaw, Poland. Email: szymon.piatek@aol.com.

\begin{abstract}
Lymph node removal as a part of surgical management is a standard of care in vulvar cancer patients. Due to patient morbidities and difficulties in treatment of inguinal healing after lymph nodes removal, lymphatic mapping has emerged as an increasingly popular option over the past few years. At this time several different techniques have been described and variety of different dyes were used. In addition, an important aspect of the use of the sentinel lymph node (SLN) technique is the removal of a limited number of lymph nodes, which allows more detailed pathological examination. Moreover, the interpretation of pathological ultra-staging of SLNs, which can identify low-volume metastases for which the clinical significance and the ideal management, remain uncertain. Despite differences in techniques and dyes used, this minimally invasive procedure is currently recommended as an alternative to full inguinofemoral lymph node dissection in selected cases by all major international societies. As for now SLN concept became a standard of care in vulvar cancer. This technique, though simple as a concept, requires a learning curve and should only be used in expert centers. This article provides a review on literature on SLN technique in vulvar cancer, current recommendations and future lines of investigation.
\end{abstract}

Keywords: Vulvar cancer; sentinel lymph node (SLN); lymphoscintigraphy; indocyanine green (ICG); review

Submitted Aug 14, 2020. Accepted for publication Nov 23, 2020.

doi: $10.21037 /$ cco-20-202

View this article at: http://dx.doi.org/10.21037/cco-20-202

\section{Introduction}

The sentinel lymph node (SLN) biopsy corresponds to a selective mapping of first lymph node which drains from a malignant tumor. This first in chain lymph node is theoretically most likely to shelter metastatic tumor cells. Detection, selectively removing and assessment of SLN can be a useful oncological procedure that minimizes morbidity related to an extensive nodal dissection in node negative patients.

The concept is not novel. Two prospective randomised trials were found proving the feasibility, reproducibility and safety of this technique $(1,2)$. Lymphatic system has been extensively studied since the seventeenth century. It has already been known already, that most cancers, epithelial in nature, metastasize via lymphatics. Virchow suggested that lymph nodes act as filters for neoplastic cells (3). This general notion was invented and incorporated in 1960 (4). Fundamental advances in the SLN biopsy was done by Cabanas, that based his work on penile carcinoma (5).

The SLN biopsy first described by Morton et al. in patients with cutaneous melanoma in 1993, has shortly after been defined in vulvar cancer $(6,7)$. Nowadays SLN is considered as a standard of care for selected cased of early stage vulvar cancer.

^ ORCID: 0000-0002-7798-3549. 


\section{Evidence for SLN in vulvar cancer}

First pilot publications by Levenback et al. and Decesare et al. confirmed that SLN in vulvar cancer is almost exclusively located in the inguinal area and can be identified in the majority of patients $(8,9)$.

On the basis of these studies two international multicentric prospective trials were opened for recruitment. The European Groningen International Study on Sentinel Nodes in Vulvar Cancer (GROINS-V) trial prospective phase II treatment trial enrolled 403 patients with unifocal, squamous cell carcinoma (SCC) confined to the vulva, tumors $<4 \mathrm{~cm}$, without suspicious nodes at palpation of the groins. All patients underwent SNL procedure, and inguinofemoral lymphadenectomy in case of metastatic SLN. The authors found a $2.3 \%$ groin recurrence rate in 259 pts with a negative SLN after a median follow up of 35 months (1). Recently published long-term results, the authors report $36.4 \%$ local recurrence rate for SLN negative. Isolated groin recurrence rate was $2.5 \%$ in SLN-negative patients at 5 years (10). For node positive patients, that received adjuvant radiotherapy, the rate of local recurrence was $46.4 \%$ and the rate of isolated groin recurrence was $8 \%$.

In the other prospective multi-institutional observational trial, GOG 173, all patients underwent SLN followed by inguinofemoral lymphadenectomy. The authors found $8.3 \%$ false negative rate of SLN and $3.7 \%$ false negative predictive value for the whole study group. However, for patients with tumors smaller than $4 \mathrm{~cm}$, the false negative predictive value was only $2 \%$ (2).

A retrospective exploratory multicenter cohort study (subgroup analysis of AGO-CaRE-1 trial) found comparable outcome for node negative patients after SLN or groin dissection (11).

The reduced morbidity is a major strong argument in favour of SLN implementation, considering that full inguinofemoral lymph node dissection leads to high rates of wound breakdown (20-40\%) and lymphedema (30-70\%). The authors of GROIN-V I study found a much lower rate of short -term complications, such as wound breakdown (11.7\% vs. $34.0 \%$, respectively; $\mathrm{P}<0.0001$ ), cellulitis ( $4.5 \%$ vs. $21.3 \%$, respectively; $\mathrm{P}<0.0001)$ when $\mathrm{SLN}$ technique was used. Long-term complications were also less frequently observed after the SLN procedure compared with inguinofemoral lymphadenectomy (erysipelas: $0.4 \% v$ s. $16.2 \%$, respectively; $\mathrm{P}<0.0001$; and lymphedema: $1.9 \%$ vs. $25.2 \%$, respectively; $\mathrm{P}<0.0001$ ).
Recently, Slomovitz et al. released the preliminary results of another prospective trial, joined work of GROINS and GOG group (GROINSV2/GOG270) (12). The study recruited a large cohort of 1,552 patients with early vulvar cancer who underwent SLN procedure only. The authors confirm the safety of omitting inguinofemoral lymphadenectomy after a negative SLN, with a groin recurrence rate of 3.1\% (after exclusion of the protocol violations $2.7 \%$ ) (12).

A systematic review and metanalysis approves the use SLN for women with unifocal tumors $<4 \mathrm{~cm}$ and clinically non-suspicious nodes in the groin (12). This large metanalysis showed that it is oncologically safe while reducing short- and long-term morbidities. The authors point out a low groin recurrence rate, comparable to standard lymph node dissection [2.8\% (1.5-4.4\%) and 1.4\% (0.5-2.6\%), respectively] and a false negative rate of SLN in order of $6.4 \%$ (4.4-8.8\%).

To summarize, the sensitivity of the technique is high and confirmed by the large prospective trials, which determines the safety. Pooled sensitivity for SLN with clinical followup for SLN-negatives is $91 \%(85-95 \%)$ with negative predictive value $95.6 \%$ (13).

\section{Technique of SLN}

Prior to surgery it is crucial to exclude bulky lymph node disease. This can be achieved using physical examination. Additional imaging (ultrasound, computerized tomography or magnetic resonance imaging) is required in obese patients in whom the sensitivity of clinical assessment is low.

The SLN technique should not be used in patients with previous groin surgery or radiation. Repeat SLN procedure in patients with recurrent vulvar cancer has been tested and found feasible. However, it is technically demanding and not yet a safe alternative to inguinofemoral LND (14).

SLN excision should be performed before the removal of the primary tumor.

The SLN procedure can be also be performed by injecting the remaining scar in patients with previous macroscopic excision of vulvar carcinoma (15).

\section{Anatomical localization of SLNs}

The vulvar SLN is localized within the inguinofemoral area. To our knowledge there is no study that reported direct lymphatic drainage from the vulva to the pelvis. Understanding of lymphatic anatomy and knowledge about localization of SLN is crucial when the technique is 
performed.

An interesting article by Rob et al. studied a detailed distribution of SLN in vulvar cancer using blue dye and $99 \mathrm{~m}$ technetium $(99 \mathrm{mTc})$ radiocolloid (16). The authors divided the lymph nodes of the groin into four regions: the superficial medial group, located above and medial to the femoral vein and medial to the saphenous vein; the superficial intermediate group, located in the vicinity of but superior and lateral to the saphenous and femoral veins; the superficial lateral group, located in the outer third of the groin; and the profundum nodes, located deep, medially along the femoral vein. The majority of SLN-58 nodes $(49.2 \%)$-was found in the superficial medial group, 41 SLNs (34.7\%) were superficial intermediate, 19 nodes (16.1\%) of SLN were deep femoral. Lymph node in deep femoral region were detected almost exclusively (17/19) in central lesions.

A group from MD Anderson evaluated patients with vulvar cancer who experienced a recurrence after undergoing lymphatic mapping and SLN biopsy. The authors observed that patients with no sentinel node identified and a negative lymphadenectomy specimen had similar patterns of recurrence. They suggest that it could be associated with nodal tissue left in the medial part of the inguinofemoral area, where the majority of SLN are found (17).

\section{Available methods of the SLN mapping}

The combination of radiotracer and blue dye has for long been gold standard in SLN mapping in vulvar cancer. This statement was based on a results of a meta-analysis which demonstrated that the SLN detection rates were $94.0 \%$ (95\% CI, 90\%-96\%) for technetium 99m Tc alone, $68.7 \%$ (95\% CI, 63\%-74\%) for blue dye alone, and 97.7\% (95\% CI, $96 \%-98 \%$ ) for $99 \mathrm{mTc}$ plus blue dye (13). In GOG 173 study, the false-negative rates were $7.8 \%$ for radiotracer alone, $2.0 \%$ for blue dye alone, and $1.6 \%$ for radiotracer and blue dye.

The radiotracer (usually technetium-99m-labeled nanocolloid) is injected in 4 cardinal points of the lesion a day prior to the surgery. The injection of a blue dye (patent blue, isosulfan blue or methylene blue) is done in the operating room setting. It is important to remember the time frames for blue dye injection. The groin can be incised 5 minutes after the injection but not later than 45 minutes, as the blue dye will no longer be visible. It is recommended to perform preoperatively a lymphoscintigraphy to show specificity of individual lymphatic pathway and facilitate the SLN procedure. The combined technique showed high (95-97.7\%) sensibility in SLN mapping (18-20).

However, its use in clinical practice may be complex, as it requires previous planning with nuclear medicine unit, the engagement of additional medical personnel, longer hospital stay and finally high costs. These inconveniences with the increasing popularity of infrared methods have contributed to the recent popularity of indocyanine green (ICG) in the identification of SLN in vulvar cancer $(21,22)$. In order to increase the fluorescence intensity and the size of the molecule, leading to a greater retention of ICG in sentinel nodes, Hutteman et al. combined ICG with a $20 \%$ albumin solution (ICG: HSA) (23). There were no differences in the effectiveness of lymph node identification using ICG and the ICG: HSA mixture. The same authors observed that all technetium-labeled SLNs were also visualized using fluorescent techniques. Verbeek et al. combined technetium isotope with ICG and demonstrated 100\% effectiveness in SLN mapping in a small group of 12 patients (24). Moreover, they found that this method allowed to reduce ICG consumption and that it could be used up to 20 hours before the surgical procedure. An alternative technique using a ferromagnet has recently been published (25). In the SARVU study, the authors showed that the use of a ferromagnet has a comparable effectiveness in detecting SLN to radiotracer.

\section{Sectioning of the lymph nodes}

The accuracy of SLN in staging vulvar cancer also depends on the technique used for histopathological examination. Postoperative pathological assessment of the SLN may be combined with intraoperative frozen section analysis. Frozen section offers the advantage of performing synchronous complete lymphadenectomy if SLN biopsy is positive. However, the opponents suggest a relatively high false negative rates and a risk of diagnostic tissue loss. Pathological examination of SLNs requires being very precise. As it was shown in other solid tumors hematoxylin and eosin staining of LN omits at least $20 \%$ of nodal metastases comparing to additional IHC examination. Additional serial sectioning was able to detect extra $7 \%$ of nodal metastases (26).

Ultrastaging of lymph nodes, using serial sectioning and immunohistochemistry, is a main focus of the SLN concept. Review by Bézu et al. showing that the rate of detection of micrometastases is significantly higher when combinating 
haematoxylin and eosin staining, with serial sectioning, and immunohistochemistry (27).

The biological significance of micrometastases detected by ultrastaging remains controversial. Isolated groin recurrence has been reported in only rare cases of patients with micrometastases in the SLN (28).

\section{Current guidelines}

The current European recommendations were published in 2017. The guidelines were supported by sufficient highlevel scientific evidence and/or by a large consensus among experts (29).

In line with current knowledge, lymph node assessment for vulvar cancer patients is indicated when tumor invasion exceeds $1 \mathrm{~mm}$. SLN procedure is recommended in patients with unifocal lesions $<4 \mathrm{~cm}$ without suspected inguinal lymph nodes at preoperative assessment preoperative (clinical groin examination and imaging).

The guidelines endorse the use of a radioactive tracer as the preferred detection method and optionally the use of a blue dye. Increased sensitivity of SLN detection is observed when both methods are used. The use of ICG has not been considered as an alternative at the time of the elaboration of guidelines.

Preoperative planar lymphoscintigraphy is recommended to allow identification, location and enumeration of lymph nodes before surgery. A sequential lymphoscintigraphy also makes it possible to differentiate the true sentinels of early appearance and the secondary lymph nodes which must not be resected.

In the absence of detection of a SLN (failure of the method), an inguinofemoral lymphadenectomy must be performed. Inguinofemoral lymphadenectomy is also required when a metastatic SLN invasion is identified.

For tumors affecting the midline (less than $1 \mathrm{~cm}$ from the midline), bilateral detection of SLN is mandatory. In case of detection a single unilateral SLN, a contralateral inguinofemoral lymphadenectomy should be performed.

As for the pathological examination, the guidelines consider SLN frozen section examination to avoid a second surgery. However, the experts' panel points the importance of a careful SLN manipulation during this procedure. This is due to tissue loss during frozen section analysis and consequently risk of omission of micrometastases on final pathological examination.

The definitive pathological exam should include an ultrastaging procedure that corresponds to serial cuts at least every $200 \mu \mathrm{m}$. If the $\mathrm{HE}$ (hematoxylin/eosin) stained sections are negative, immunohistochemistry analysis should be performed.

\section{Metastatic SLN}

Metastatic lymph node disease in early vulvar cancer is diagnosed in $10.7 \%$ of stage I and $26.2 \%$ of stage II vulvar cancer $21-35.8 \%$ of patients (30). Lymph node metastasis is definitely associated with poorer disease-free and overall survival. Mahner et al. report disease-free survival 35.2\%, and the overall survival rate $56.2 \%$ compared with $75.2 \%$ and $90.2 \%$ in node negative patients (31).

\section{The volume of lymph node disease and survival}

The lymph node metastases are classified as stage III disease. Fédération Internationale de Gynécologie et d'Obstétrique (FIGO) classification considers not only the presence and count of metastatic lymph nodes $(\leq 2$ and $>2$ lymph nodes) but also the volume of lymph node disease $(<5$ and $\geq 5 \mathrm{~mm}$ ). These subclassification is based on the results of GROINS-V trial. Oonk et al. showed that size of SLN metastases is related with the affectation of other lymph nodes (32). The risk of founding a metastatic lymph node other than SLN is increased when the volume of disease in the SLN is higher. Moreover, the authors showed differences in 2 years disease specific survival between patients with SLN metastases $\leq 2$ and $>2 \mathrm{~mm}(69.5 \%$ vs. 94.4\% respectively, $\mathrm{P}=0.001$ ).

\section{Management of metastatic groin nodes}

Management of patients with metastatic groins is controversial. The current guidelines recommend inguinofemoral lymph node dissection at any size of SLN metastasis and the use of adjuvant therapy in case of extracapsular disease or presence of metastases in more than one lymph node. There are no specifically established recommendations for the management of micrometastases and isolated tumor cells.

The role of adjuvant treatment in node-positive disease was studied in a large multicenter retrospectives study of the AGO group. The authors reported three-year PFS and OS rates improvement when radiotherapy was used in these patients compared with node-positive patients without adjuvant treatment (31).

This problem was also addressed in prospective trials. 
In GROINSS-V II, a prospective multicentre phase II trial, patients with early-stage SCC of the vulva (diameter $<4 \mathrm{~cm}$ ) without suspicious lymph nodes at imaging, who had primary surgery with SNL procedure were included. In case of a metastatic $\mathrm{SN}$ (metastasis of any size), radiotherapy was given to the groin(s) (50 Gy). In case of a negative SN, patients were followed-up for $\geq 2$ years. Stopping rules were defined for both groups to monitor groin recurrence rate. The authors concluded that for patients with $\mathrm{SN}$ metastasis $>2 \mathrm{~mm}$, radiotherapy with a total dose of 50 Gy was no safe alternative. However, adjuvant therapy can be a safe alternative for inguinofemoral lymphadenectomy in patients with SLN metastasis $\leq 2 \mathrm{~mm}$, with minimal toxicity (33).

Currently, a GROIN-V III study that evaluates the role of chemoradiation in patients with metastatic SLN is ongoing.

\section{Conclusions}

At present, SLN biopsy in early stage vulvar cancer has been adopted in the majority of Gynecologic Oncology Units $(34,35)$. However, it must be stressed that its use requires a learning curve, argument to centralize the surgical management of vulvar cancer in specialized centers with appropriate facilities (nuclear medicine or ICG fluorescence equipment).

Although isolated groin recurrence is a rare event, its prognosis is uncertain. The median survival after diagnosis of the nodal recurrence is described to be in order of 9 months (range, 3-30 months) with only limited number of patients having long-term survival $(36,37)$. Considering the poor prognosis of nodal recurrence, improper use of the SLN technique can be detrimental for our patients.

SLN procedure showed excellent results in management of early stage vulvar cancer. However, it should be used wisely and only in setting of multidisciplinary expert team.

\section{Acknowledgments}

Funding: None.

\section{Footnote}

Provenance and Peer Review: This article was commissioned by the Guest Editors (Denis Querleu and Cherif Youssef Akladios) for the series "Sentinel Lymph Node Biopsy in Gynecologic Cancer" published in Chinese Clinical Oncology. The article was sent for external peer review organized by the Guest Editors and the editorial office.
Conflicts of Interest: All authors have completed the ICMJE uniform disclosure form (available at http://dx.doi. org/10.21037/cco-20-202). The series "Sentinel Lymph Node Biopsy in Gynecologic Cancer" was commissioned by the editorial office without any funding or sponsorship. The authors have no other conflicts of interest to declare.

Ethical Statement: The authors are accountable for all aspects of the work in ensuring that questions related to the accuracy or integrity of any part of the work are appropriately investigated and resolved.

Open Access Statement: This is an Open Access article distributed in accordance with the Creative Commons Attribution-NonCommercial-NoDerivs 4.0 International License (CC BY-NC-ND 4.0), which permits the noncommercial replication and distribution of the article with the strict proviso that no changes or edits are made and the original work is properly cited (including links to both the formal publication through the relevant DOI and the license). See: https://creativecommons.org/licenses/by-nc-nd/4.0/.

\section{References}

1. Van der Zee AG, Oonk MH, De Hullu JA, et al. Sentinel node dissection is safe in the treatment of early stage vulvar cancer. J Clin Oncol 2008;26:884-9.

2. Stehman FB, Ali S, DiSaia PJ. Node count and groin recurrence in early vulvar cancer: a gynecologic oncology group study. Gynecol Oncol 2009;113:52-6.

3. Weinberg J, Greaney EM. Identification of regional lymph nodes by means of a vital staining dye during surgery of gastric cancer. Surg Gynecol Obstet 1950;90:561-7.

4. Gould EA, Winship T, Philbin PH, et al. Observations on a "sentinel node" in cancer of the parotid. Cancer 1960;13:77-8.

5. Cabanas RM. An approach for the treatment of penile carcinoma. Cancer 1977;39:456-66.

6. Morton DL, Wen DR, Foshag LJ, et al. Intraoperative lyphatic mapping and selective cervival lymphadenectomy for early stage melanomas of the head and neck. J Clin Oncol 1993;11:1751-6.

7. Levenback C, Burke TW, Gershenson DM, et al. Intraoperative lymphatic mapping in vulvar cancer. Obstet Gynecol 1994;84:163-7.

8. Levenback C, Burke TW, Morris M, et al. Potential applications of intraoperative lymphatic mapping in vulvar cancer. Gynecol Oncol 1995;59:216-20. 
9. Decesare SL, Fiorica JV, Roberts WS, et al. A pilot study utilizing intraoperative lymphoscintigraphy for identification of the sentinel lymph nodes in vulvar cancer. Gynecol Oncol 1997;66:425-8.

10. Te Grootenhuis NC, van der Zee AG, van Doorn HC, et al. Sentinel nodes in vulvar cancer: Long-term followup of the GROningen INternational Study on Sentinel nodes in Vulvar cancer (GROINSS-V) I. Gynecol Oncol 2016;140:8-14.

11. Klapdor R, Hertel H, Soergel P, et al. Application of sentinel lymph node dissection in gynecological cancers: results of a survey among German hospitals. Arch Gynecol Obstet 2017;295:713-20.

12. Slomovitz B, Oonk M, Monk BJ, et al. Validation of sentinel lymph biopsy in patients with early stage vulvar cancer: A prospective trial of 1552 women (GROINSV2/ GOG270). Submitted to: Society of Gynecologic Oncology (SGO) Annual Meeting on Women's Cancer. 2020;Abstract 2.

13. Covens A, Vella ET, Kennedy EB, et al. Sentinel lymph node biopsy in vulvar cancer: Systematic review, metaanalysis and guideline recommendations. Gynecol Oncol 2015;137:351-61.

14. van Doorn HC, van Beekhuizena HJ, Gaarenstroom KN. Repeat sentinel lymph node procedure in patients with recurrent vulvar squamous cell carcinoma is feasible. Gynecol Oncol 2016;140:415-9.

15. Nica A, Covens A, Vicus D, et al. Long-term outcomes in patients with sentinel lymph nodes (SLNs) identified by injecting remaining scar after previously excised vulvar cancer. Gynecol Oncol 2019;155:83-7.

16. Rob L, Robova H, Pluta M, et al. Further data on sentinel lymph node mapping in vulvar cancer by blue dye and radiocolloid Tc99. Int J Gynecol Cancer 2007;17:147-53.

17. Frumovitz M, Ramirez PT, Tortolero-Luna G, et al. Characteristics of recurrence in patients who underwent lymphatic mapping for vulvar cancer. Gynecol Oncol 2004;92:205-10.

18. Meads C, Sutton AJ, Rosenthal AN, et al. Sentinel lymph node biopsy in vulval cancer: systematic review and metaanalysis. Br J Cancer 2014;110:2837-46.

19. Hassanzade M, Attaran M, Treglia, G, et al. Lymphatic mapping and sentinel node biopsy in squamous cell carcinoma of the vulva: systematic review and metaanalysis of the literature. Gynecol Oncol 2013;130:237-45.

20. Lawrie TA, Patel A, Martin-Hirsch PPL et al. Sentinel node assessment for diagnosis of groin lymph node involvement in vulval cancer. Cochrane Database Syst Rev
2014(6):CD010409

21. Crane LM, Themelis G, Arts HJ, et al. Intraoperative near-infrared fluorescence imaging for sentinel lymph node detection in vulvar cancer: first clinical results. Gynecol Oncol 2011;120:291-5.

22. Schaafsma BE, Verbeek FP, Peters AA, et al. Near-infrared fluorescence sentinel lymph node biopsy in vulvar cancer: a randomised comparison of lymphatic tracers. BJOG 2013;120:758-64.

23. Hutteman M, van der Vorst J, Gaarenstroom K, et al. Optimization of near-infrared fluorescent sentinel lymph node mapping for vulvar cancer. Am J Obstet Gynecol 2012;206:89.e1-5.

24. Verbeek FP, Tummers QR, Rietbergen DD, et al. Sentinel Lymph Node Biopsy in Vulvar Cancer Using Combined Radioactive and Fluorescence Guidance. Int J Gynecol Cancer 2015;25:1086-93.

25. Jedryka M, Kryszpin M, Manowiec M, et al. A new technique of sentinel lymph nodes detection in vulvar cancer patients. The SARVU study. Int J Gynecol Cancer 2019:29:A1-A197.

26. Cote RJ, Fpeterson H, Chaiwun B, et al. Role of immunohistochemical detection of lymph-node metastases in management of breast cancer. Lancet 1999;354:896-900.

27. Bézu C, Coutant C, Ballester M, et al. Ultrastaging of lymph node in uterine cancers. J Exp Clin Cancer Res 2010;29:5.

28. Tamussino KF, Bader A, Lax S, et al. Groin recurrence after micrometastasis in a sentinel lymph node in a patient with vulvar cancer. Gynecol Oncol 2002;86:99-101.

29. Oonk MHM, Planchamp F, Baldwin P, et al. European Society of Gynaecological Oncology guidelines for the management of patients with vulvar cancer. Int J Gynecol Cancer 2017;27:832-7.

30. Berek J, Hacker N. editors. Practical Gynecologic Oncology, 3rd ed. Baltimore: Lippincott Williams \& Wilkins, 2000.

31. Mahner S, Jueckstock J, Hilpert F, et al. Adjuvant Therapy in Lymph Node-Positive Vulvar Cancer: The AGOCaRE-1 Study. J Natl Cancer Inst 2015;107:dju426.

32. Oonk MH, van Hemel BM, Hollema H, et al. Size of sentinel-node metastasis and chances of non-sentinelnode involvement and survival in early stage vulvar cancer: results from GROINSS-V, a multicentre observational study. Lancet Oncol 2010;11:646-52.

33. Oonk M, Slomovitz B, Baldwin P, et al. Radiotherapy instead of inguinofemoral lymphadenectomy in vulvar 
cancer patients with a metastatic sentinel node: results of GROINSS-V II. Int J Gynecol Cancer 2019;29:A14.

34. Rottmann M, Beck T, Burges A, et al. Trends in surgery and outcomes of squamous cell vulvar cancer patients over a 16-year period (1998-2013): a population-based analysis. J Cancer Res Clin Oncol 2016;142:1331-41.

35. Cham S, Chen L, Burke WM, et al. Utilization and Outcomes of Sentinel Lymph Node Biopsy for Vulvar

Cite this article as: Rychlik A, Bidzinski M, Rzepka J, Piatek S. Sentinel lymph node in vulvar cancer. Chin Clin Oncol 2021;10(2):19. doi: 10.21037/cco-20-202
Cancer. Obstet Gynecol 2016;128:754-60.

36. Cormio G, Loizzi V, Carriero C, et al. Groin recurrence in carcinoma of the vulva: management and outcome. Eur J Cancer Care (Engl) 2010;19:302-7.

37. Frey JN, Hampl M, Mueller MD, et al. Should Groin Recurrence Still Be Considered as a Palliative Situation in Vulvar Cancer Patients?: A Brief Report. Int J Gynecol Cancer 2016;26:575-9. 